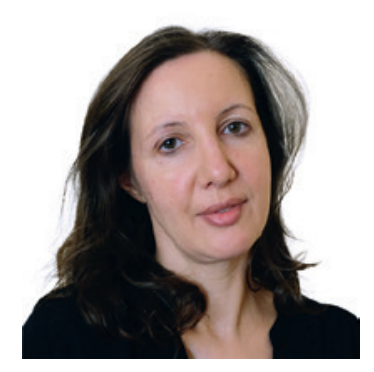

\title{
Nutritional Therapy in COVID-19 Management
}

Cecilia Medeiros de Morais

Faculty of Nutrition and Food Sciences, University of Porto, Porto, Portugal

Abstract from Fernández-Quintela A, Milton-Laskibar I, Trepiana J, et al.: Key aspects in nutritional management of COVID-19 patients. J Clin Med. 2020;9(8):2589.

\section{Keywords}

COVID-19 · SARS-CoV-2 · Bioactive compounds · Malnutrition . Nutrition · Nutritional support

\begin{abstract}
This review deals with the relationship among nutrition, the immune system, and coronavirus disease 2019 (COVID-19). The influence of nutrients and bioactive molecules present in foodstuffs on immune system activity, the influence of COVID-19 on the nutritional status of the patients, and the dietary recommendations for hospitalized patients are addressed. Deficient nutritional status is probably due to anorexia, nausea, vomiting, diarrhea, hypoalbuminemia, hypermetabolism, and excessive nitrogen loss. There is limited knowledge regarding the nutritional support during hospital stay of COVID-19 patients. However, nutritional therapy appears as first-line treatment and should be implemented
\end{abstract}

into standard practice. Optimal intake of all nutrients, mainly those playing crucial roles in immune system, should be assured through a diverse and well-balanced diet. Nevertheless, in order to reduce the risk and consequences of infections, the intakes for some micronutrients may exceed the recommended dietary allowances since infections and other stressors can reduce micronutrient status. In the case of critically ill patients, recently published guidelines are available for their nutritional management. Further, several natural bioactive compounds interact with the angiotensin-converting enzyme 2 (ACE2) receptor, the gateway for severe acute respiratory syndrome (SARS) and severe acute respiratory syndrome coronavirus 2 (SARS-CoV-2). Natural bioactive compounds can also reduce the inflammatory response induced by SARS-CoV-2. These compounds are potential beneficial tools in the nutritional management of COVID-19 patients.

(c) 2020 The Authors 


\section{Knowledge Transfer}

\section{Background}

Since the first cases of coronavirus disease 2019, the society has been challenged to live in a new reality. Considered a high threat to human health, this illness caused by the severe acute respiratory syndrome coronavirus 2 (SARS-CoV-2) causes infection in the respiratory system. At cell level, SARS-CoV-2 binds to angiotensin-converting enzyme 2 (ACE2) receptors causing an inflammatory response. As a consequence, infected individuals may remain asymptomatic or present a variety of moderate to more severe symptoms. Among those symptoms are some that directly or indirectly affect dietary intake and nutrient or energy requirements such as fever, fatigue, dry cough, anosmia/dysgeusia, pneumonia with dyspnoea, and also muscle and joint pain, headache, diarrhoea, nausea or vomiting. This study provides research on nutrients and dietary compounds that affect the immune system and interact with the ACE2 receptors, as well as the ones that are important for the treatment of COVID-19 patients [1, 2].

\section{Study Results}

In summary, high biological value proteins, fatty acids (omega 3), vitamins A and C, dietary fibre, selenium and copper present anti-inflammatory effects; polar lipids have an anti-thrombotic effect; vitamins $A, C$ and D protect against respiratory infections; vitamin $E$, iron and zinc improve the immune function; and vitamins $C, A$ and $E$, and omega 3 fatty acids present antioxidant effects (Fig. 1). Consumption of carbohydrates with a higher glycaemic index should be avoided since this contributes to inflammation. Supplementation is required when the recommended dietary intake is compromised but remains controversial for patients without deficiency. Chronic diseases, malnutrition, or COVID-19 itself can compromise meeting nutritional needs. Obesity and excessive adiposity, but especially low muscle mass are risk factors for COVID-19 patients. Professionals should analyse cases individually and search for signs of deficiency or environmental factors that may influence the nutritional needs. Natural bioactive compounds found in plants and sea food, namely revesterol, eicosapentaenoic acid (EPA) and docosahexaenoic acid (DHA), capsaicin, and curcumin, among others, have been associated with anti-inflammatory effects. Nevertheless, the administration of those components requires more research. At last, considering the important connection between gut microbiota and immunity, probiotics and prebiotics revealed a protective effect and are promising compounds for a dietary therapy of COVID-19 patients [1, 3].

\section{Nutritional Status and Therapy}

Given the fact that COVID-19 particularly affects the older age groups who are already at risk for malnutrition, it is recommended

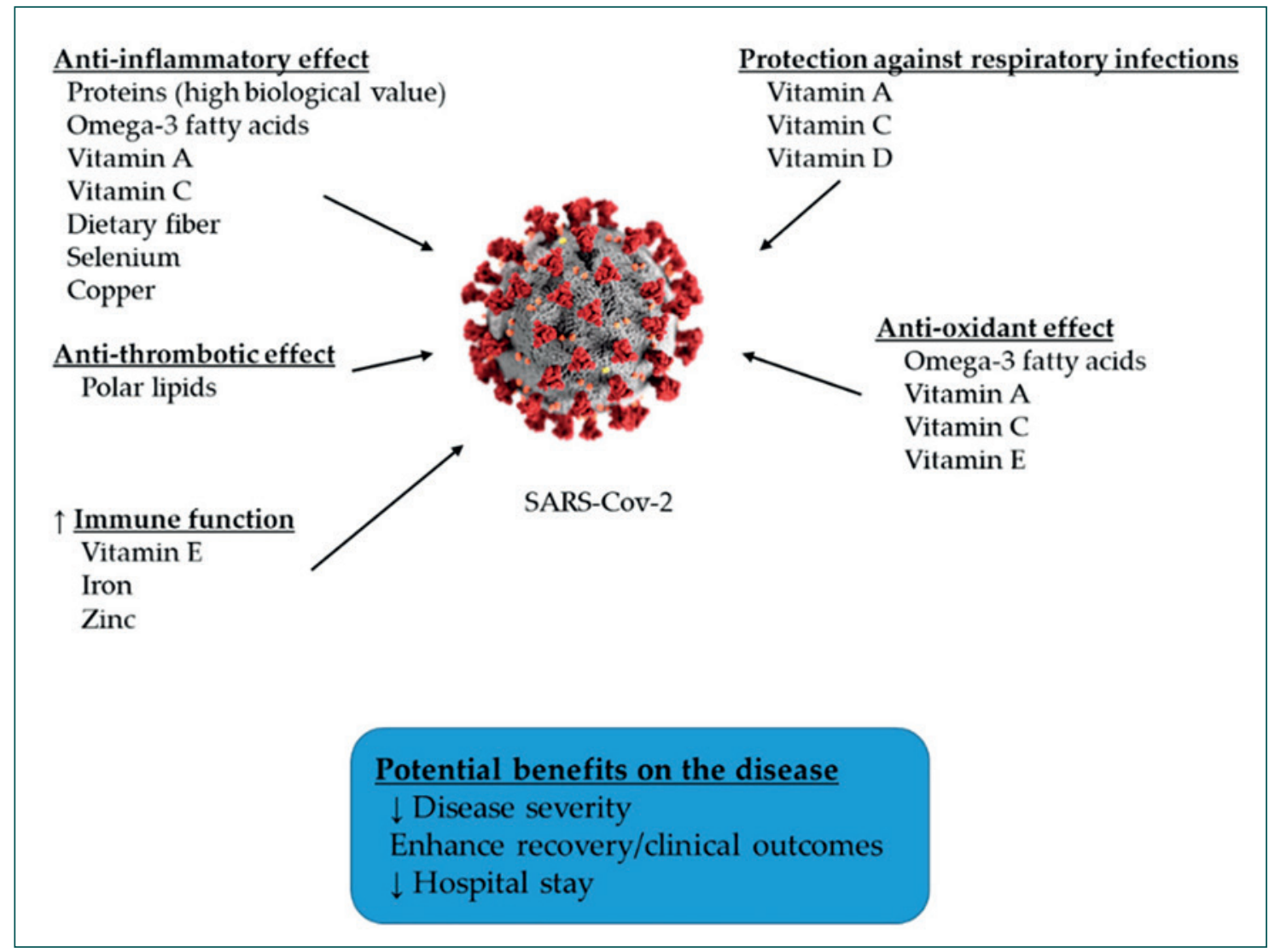

Fig. 1. Effects of several nutrients on aspects of COVID-19 infection [1]. 
to proceed with the screening and assessment of nutritional status using standardized tools. Environmental and personal conditions (palatability of food, swallowing difficulties, low mobility of the gastrointestinal tract, dysgeusia, etc.) can also compromise nutritional status $[4,5]$. Enteral and parenteral nutrition should be considered whenever oral feeding is not possible. Non-critically ill patients should follow a healthy diet and supplements of some nutrients as vitamins $D$ and $E$ and folates can be used [1, 3]. Formulas rich in protein and low in glucose are used for enteral or parenteral feeding. For critically ill patients, experts recommend a hypocaloric diet in the first week. However, long-term patients should not consume hyper- or hypocaloric diets. In these cases, supplementation in micronutrients is required [1].

\section{Conclusion}

Studying dietary components and specific bioactive compounds for the management of COVID-19 is extremely important and should focus on the changes in nutrient requirements and the impact on the immune systems of patients from different age groups. Nutritional therapy is an essential component for the successful treatment and recovery of COVID-19 patients.

\section{Disclosure Statement}

I hereby declare that there are no conflicts of interest with regard to this commentary.

\section{References}

1 Fernández-Quintela A, Milton-Laskibar I, Trepiana J, et al.: Key aspects in nutritional management of COVID-19 patients. J Clin Med. 2020;9(8):2589.

2 Lisi L, Lacal PM, Barbaccia ML, et al.: Approaching coronavirus disease 2019: Mechanisms of action of repurposed drugs with potential activity against SARS-CoV-2. Biochem Pharmacol. 2020;180:114169.

3 Laviano A, Koverech A, Zanetti M: Nutrition support in the time of SARSCoV-2 (COVID-19). Nutrition. 2020;74:110834.

4 Cervantes-Pérez E, Cervantes-Guevara G, Martínez-Soto Holguín MC, et al.: Medical nutrition therapy in hospitalized patients with SARS-CoV-2 (COVID-19) infection in a non-critical care setting: knowledge in progress. Curr Nutr Rep. 2020;DOI:10.1007/s13668-020-00337-x.

5 Calder PC, Carr AC, Gombart AF, et al.: Optimal nutritional status for a wellfunctioning immune system is an important factor to protect against viral infections. Nutrients. 2020;12(4):1181.

Correspondence: Cecilia Medeiros de Morais, PhD, Faculty of Nutrition and Food Sciences, University of Porto, R. Dr. Roberto Frias S/N, 4200-465 Porto, Portugal, cecilia_mmorais@hotmail.com 